\title{
A Taguchi and Neural Network Based Electric Load Demand Forecaster
}

\author{
Albert W. L. Yao ${ }^{1, *}$, H. T. Liao ${ }^{2}$ and C. Y. Liu ${ }^{3}$ \\ ${ }^{I}$ Department of Mechanical and Automation Engineering, National Kaohsiung First University of Science and Tech- \\ nology, Kaohsiung. ${ }^{2}$ Department of Mechanical Engineering, Minghsin University of Science and Technology, \\ Hsinchu. ${ }^{3}$ Department of Mechanical Engineering, Southern Taiwan University, Tainan, Taiwan, R.O.C.
}

\begin{abstract}
In this paper, we present Taguchi's and rolling modeling methods of artificial neural network (ANN) for very-short-term electric demand forecasting (VSTEDF) from the consumers' viewpoint. The rolling model is a metabolism technique that guarantees input data are always the most recent values. In ANN prediction, several factors that may influence the model should be well examined. Taguchi's method was employed to optimize the parameter settings for the ANN-based electric demand-value forecaster. Our experimental result shows that the optimal settings of ANN prediction model are 3 lagged load points, 0.1 for the momentum, 5 hidden neurons and 0.1 for the learning rate. The error of forecasting is as small as 3\%. That is, comparison with the results of ordinary ANN and Grey prediction, the presented Taguchi-ANN-based forecaster gives more accurate prediction for VSTEDF.
\end{abstract}

\section{INTRODUCTION}

Due to the lack of natural energy resources, over 95 percent of the energy consumed in Taiwan is imported from overseas, and owing to the growth of the economy and global market competition, the supply and demand of high-quality and inexpensive electric power has become an important issue to electric power plants, business owners, and government. In order to assess electricity efficiently and supply high-quality electricity to the consumers economically, electric power companies face financial and technical challenges. Tracking electric load generation at all times and knowledge of the future load is a basic requisite in the efficient operation of power-generating facility.

In the past few decades, numerous researchers have presented different methods for electric load forecasting. Among these studies, many models for load forecasting have been proposed, such as models of time-series analysis, regression analysis, artificial neural networks (ANN) and Grey prediction. These models have been reported and documented demonstrating success in long-term forecasting, medium-term forecasting, short-term forecasting and very-short-term forecasting.

A number of researchers have adopted ANN for load forecasting. Fung and Tummala (Fung 1993) [1] combined the economic factors such as electricity price, gross domestic product, and the weather conditions to predict the long-term load consumption for each electricity market in Hong Kong. Charytoniuk and Chen (Charytoniuk 2000) [2] moved their focus toward predicting relative changes in a daily load base. Instead of modeling relationships between load, time, weather conditions, they extrapolated the recently observed load patterns to the nearest future. Srinivasan et al. (Srinivasan 1991) [3] used a three-layer-architecture back-propagation networks model (BPN) to predict

\footnotetext{
"Address correspondence to this author at the Department of Mechanical and Automation Engineering, National Kaohsiung First University of Science and Technology, Kaohsiung; Taiwan; E-mail: yao@exchange.nkfust.edu.tw
}

the daily peak load in Singapore. They conclude that using a lower learning rate for the layers gives accurate prediction. Lu et al. (Lu 1993) [4], Kiartzis (Kiartzis 1995) [5], Khotanzad (Khotanzad 1998) [6], Chow (Chow 1996) [7] and Bakirtzis (Bakirtzis 1996) [8] used different BPN models to predict hourly load, daily total load and daily peak load. They also show that there is no firm criterion for training inputs selection.

However, the load demand forecasting from the viewpoint of consumers is seldom discussed. With global marketing competition, national and multinational enterprises have tried very hard to cut down on their expenditures in order to increase their competitiveness. Additionally, power companies generally have many different rate schedules and penalty policies of poor-power-factor loads for their customers. Therefore, the practice of demand-control of electric systems has drawn a lot of attention from government, manufacturers and researchers.

Likewise, the electric bill is always one of the largest monthly bills in educational institutes. Yao and $\mathrm{Ku}$ (Yao 2003) [9] developed and installed a cost-effectively network-based and PC-based automated monitoring and control electric system (AMCES) at the National Kaohsiung First University of Science and Technology (NKFUST), Taiwan, to monitor and control the electric load on campus. AMCES (see Fig. 1) is a closed-loop demand-control system with intelligent predictor embedded and networked with a PC-based Human-Machine Interface and Data Acquisition system (HMI/DAS). AMCES provides an effective solution to electricity management.

The aim of this project is to design a dynamic forecaster for VSTEDF. We present a Taguchi-rolling-model-based method for establishing the training data of ANN to overcome the deficiency of BPN on VSTEDF. This paper is organized as follows. Section 2 shows our preliminary forecasting results using ordinary BPN. Section 3 presents the development of a rolling model of BPN for VSTEDF. Section 4 presents the results and discussion. Section 5 ends with the conclusions of this study. 


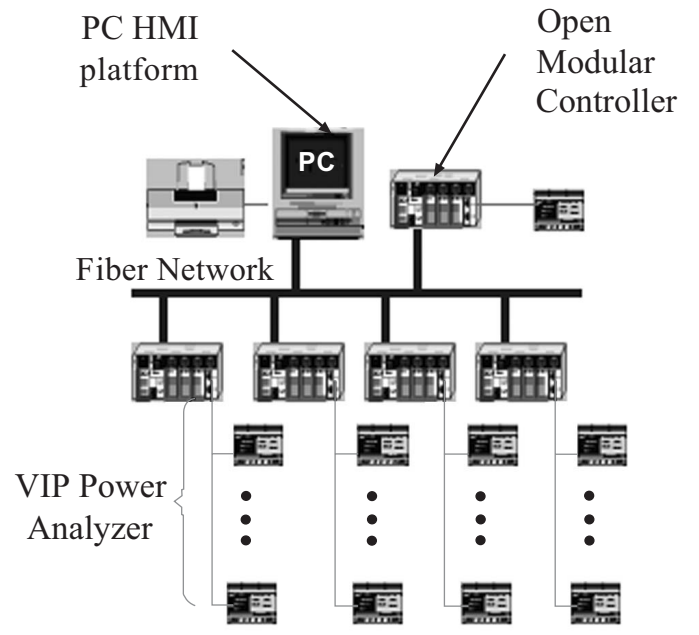

Fig. (1). Configuration of AMCES and its components. It is based on PC-based OMC, a PC-based visual HMI/DAS and digital power analyzers.

\section{PRELIMINARY FORECASTING BY CNVENTIONAL BPN}

The past research activities of ANN-based load forecasting often involved the daily peak load or total load with the factors of temperature, seasons, or day-types. Generally, a typical ANN architecture for electric load forecasting is shown in Fig. 2. It generally includes temperature, electric load and day-types as inputs for ANN training. The selection of input variables for ANN training always plays an important role in the accuracy of prediction. The model of ANN is establishing the relationships between the inputs and outputs through the training operation process. Generally, if the inputs represent less related to the predicting output, ANN may not be expected to accurately predict the outputs (Drezga 1998) [10]. However, there are no general rules for selecting input variables. Efficiently selecting appropriate variables depends on experience or preliminary tests (Swarup 2002) [11].

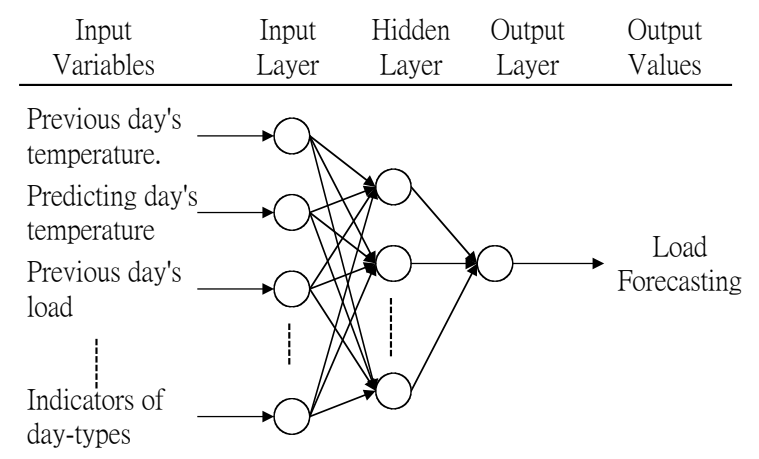

Fig. (2). A typical ANN architecture for electric load forecasting. It often includes the temperature, load, and the day-types as training inputs for the neural network model.

In our preliminary study, we adopted ordinary BPN model and considered the electric loads and the temperatures of the last 2 minutes and 4 minutes, and the temperature of the next 2 minutes as the training inputs for the BPN. Then, using this established model to forecast and examine the electric load for the next 2 minutes (see Table 1). The
Table 1. The Description of Inputs for VSTEDF by Using the Ordinary BPN Model. Input Nodes 1 and 2 are loads of the Last $2 \mathrm{~min}$. and $4 \mathrm{~min}$. Input Nodes 3 and 4 are the Temperatures of the Last $2 \mathrm{~min}$. and $4 \mathrm{~min}$. Input node 5 is the Temperature of the Next $2 \mathrm{~min}$. The Output Node is the Load Prediction

\begin{tabular}{|c|c|}
\hline Input Node & Variables \\
\hline \hline $1 \& 2$ & Load $(\mathrm{M}-i), i=2,4$, \\
\hline $3 \& 4$ & Temperature $(\mathrm{M}-i), i=2,4$, \\
\hline 5 & Temperature for the next 2 minutes $(\mathrm{M}+2)$ \\
\hline Output node & Forecasted load \\
\hline \multicolumn{2}{|c|}{ Note: $\mathrm{M}=$ Minute } \\
\hline
\end{tabular}

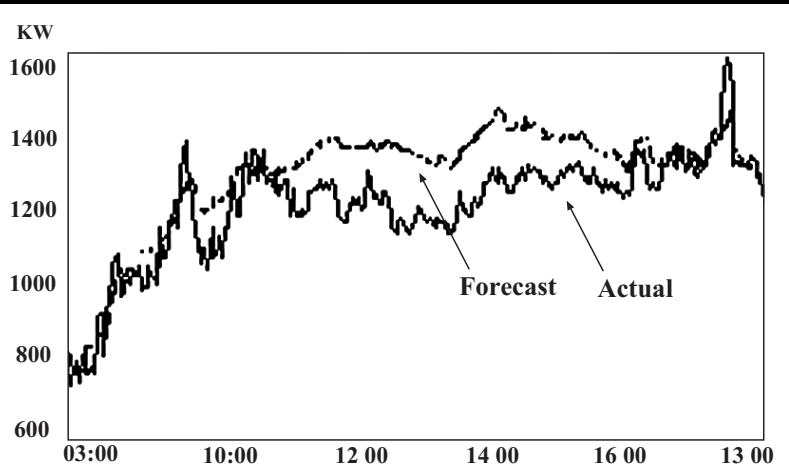

Fig. (3). Preliminary forecasting results by conventional BPN model which with electric load and temperature as the input variables of networks shows that a conventional BPN model has a poor result for VSTEDF.

forecasted electric demand values of ordinary BPN are shown in Fig. 3. The total averaged absolute error is about $14 \%$. It concludes that the ordinary BPN model does not deal adequately with a volatile system like VSTEDF. The volatility of the usage of electricity on NKFUST's campus is possibly due to the load characteristics of lamps, motors, and air-conditioning varying according to the class schedule and the operation of equipment. Weather related effects are also significant factors. Heat waves and humidity in the southern Taiwan are major factors of the influence of electric load in summer. Specifically, the electric load for lighting and air-conditioning is highly influenced by the temperature and the presence of clouds. However, the other uncontrolled factors of the electric load of the indoor activity are the class schedule, the attendance of students, laboratory hours, and so on. The pattern of electric load consumption is not always regular in the daily usage of the electricity on campus.

From the preliminary study, the distribution plot of the load versus the temperature is shown in Fig. 4. As can be seen, it indicates that the factor of temperature is unrelated with the electric load. Therefore we considered the relationship of electric load and temperature as a relatively large time constant and ignored the temperature input in our study. But we trained our ANN model with the pattern recognition of temperature. The procedures are giving as follows. 


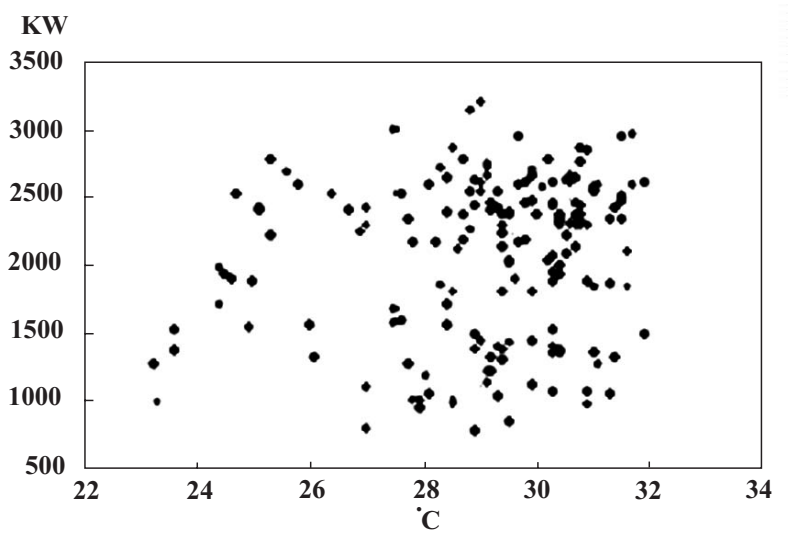

Fig. (4). Distribution plot of electric load versus temperature. It shows that the electric load is unrelated to the temperature.

\section{ROLLING MODEL OF BPN FOR VSTEDF}

\section{Rolling Model of BPN Input Patterns}

The general BPN-based load forecasting is a kind of non-linear regression model, which is derived based on the functional relationship between the lagged values of the time series inputs and several reasonable explanatory variables (Makridakis 1998) [12]. In VSTEDF, it is very difficult to decide the affecting factors owing to the volatile change of electric load. However, the electric load is a form of time series data. The models of traditional methods for time series analysis involve the methods of moving average (MA), auto-regression (AR), or the combination of the two, the ARMA model (Bose 1996) [13]. In the AR model, it sums up the weighted $n$ immediate past values of $y_{i}$ and the weighted value of the present input to predict the current value of the time-series. That is,

$$
y_{i}=\sum_{j=1}^{n} a_{j} y_{i-j}=a_{1} y_{i-1}+a_{2} y_{i-2}+\mathrm{L}+a_{n} y_{i-n}
$$

However, it takes a lot of effort to solve the coefficient $a_{j}$ by the least square method in the AR model. Unlike AR modeling, the ANN model only needs several historical input variables to train the ANN for predicting the focused output. Moreover, the Grey prediction often adopts rolling model technique, which utilizes the forward data points in the same series to construct Grey model for predicting the next data (Yao 2003) [14]. It leads us to the idea of constructing a rolling model technique for BPN of VSTEDF. The rolling model in Fig. 5 is a metabolism technique that

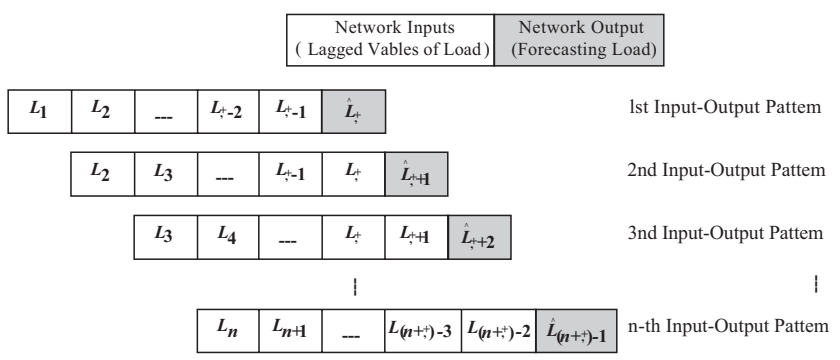

Fig. (5). Rolling model of input pattern of BPN. It refreshes a new input data by dropping the oldest data. updates the input data by discarding the oldest data for each cycling time. The rolling modeling mechanism provides a means to guarantee input data are always the most recent values.

The typical development of the rolling model of BPN for the VSTEDF is constructed as follows. For instance, an AI forecasting model consists of one hidden layer with two hidden neurons $\left(\mathrm{H}_{1}\right.$ and $\left.\mathrm{H}_{2}\right)$, and $\mathrm{n}$ input neurons $\left(\mathrm{x}_{1}, \ldots, \mathrm{x}_{\mathrm{n}}\right)$ (see Fig. 6). The formulae of the model are listed in (2) to (5).

$$
\begin{array}{r}
h_{1}=f_{1}\left(x_{1}, x_{2}, \mathrm{~L}, x_{n}\right)=f_{1}\left(L_{1}, L_{2}, \mathrm{~L}, L_{n}\right) \\
=\frac{1}{1+\exp \left[-\left(w_{11} L_{1}+w_{12} L_{2}+\mathrm{L}+w_{1 n} L_{n}-\theta_{1}\right)\right]} \\
h_{2}=f_{2}\left(x_{1}, x_{2}, \mathrm{~L}, x_{n}\right)=f_{2}\left(L_{1}, L_{2}, \mathrm{~L}, L_{n}\right) \\
=\frac{1}{1+\exp \left[-\left(w_{21} L_{1}+w_{22} L_{2}+\mathrm{L}+w_{2 n} L_{n}-\theta_{2}\right)\right]} \\
y=\hat{L}_{n+1}=f_{3}\left(h_{1}, h_{2}\right) \\
=\frac{1}{1+\exp \left[-\left(w_{46} h_{1}+w_{56} h_{2}-\theta_{3}\right)\right]} \\
\Rightarrow \hat{L}_{n+1}=f\left(L_{1}, L_{2}, \ldots, L_{t}\right)
\end{array}
$$

where

$$
\begin{aligned}
& x=\text { Input variable of BPN } \\
& y=\text { Output variable of BPN } \\
& \hat{L}_{t}=\text { Forecasting load at time } t \\
& L=\text { Historical values of load } \\
& w=\text { Weights of ANN } \\
& \theta=\text { Bias of ANN } \\
& h=\text { Signal flow of hidden neurons }
\end{aligned}
$$

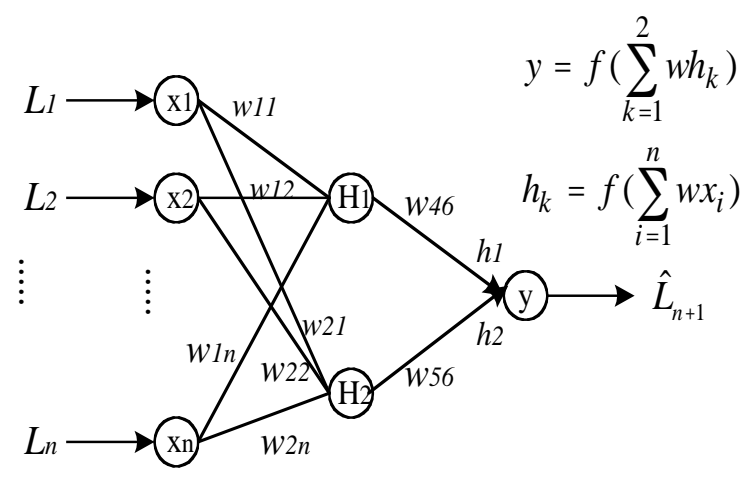

Fig. (6). A typical BPN forecasting model consists of one hidden layer with two hidden neurons $\left(\mathrm{H}_{1}\right.$ and $\left.\mathrm{H}_{2}\right)$, and $\mathrm{n}$ input neurons $\left(\mathrm{x}_{1}, \ldots, \mathrm{x}_{\mathrm{n}}\right)$. The BPN algorithm can establish the non-linear relationship between the forecasted demand and lagged values of load. The $y$ is output variable of BPN and the $h_{k}$ is signal flow of hidden neurons.

The forecasting model can be described by (5) $\hat{L}_{n+1}=f\left(L_{1}, L_{2}, \ldots, L_{t}\right)$, where $L_{t}$ denotes the set of lagged load inputs. 


\section{Planning and Implementation of Training Process for VSTEDF}

The process of model training is the most important task of the application of ANN. The applicability of training data will seriously affect the prediction of the ANN-based forecaster. The selection of some other parameters of ANN are also very important to the predictor's performance, such as learning rate, numbers of hidden layers and neurons, and momentum, which need to be decided efficiently and scientifically.

According to the historical data of electric load consumption on campus, the usage pattern of electricity was identified and analyzed. The proposed flowchart of training process for VSTEDF is shown in Fig. 7. First, the selection rule of training data has to be chosen. Then, the normalization of the training data has to be done because of the limitation of BPN's transfer function. We employed Taguchi's experimental method as a means to obtain the optimal parameter settings of BPN quickly. The procedures are giving as follows.

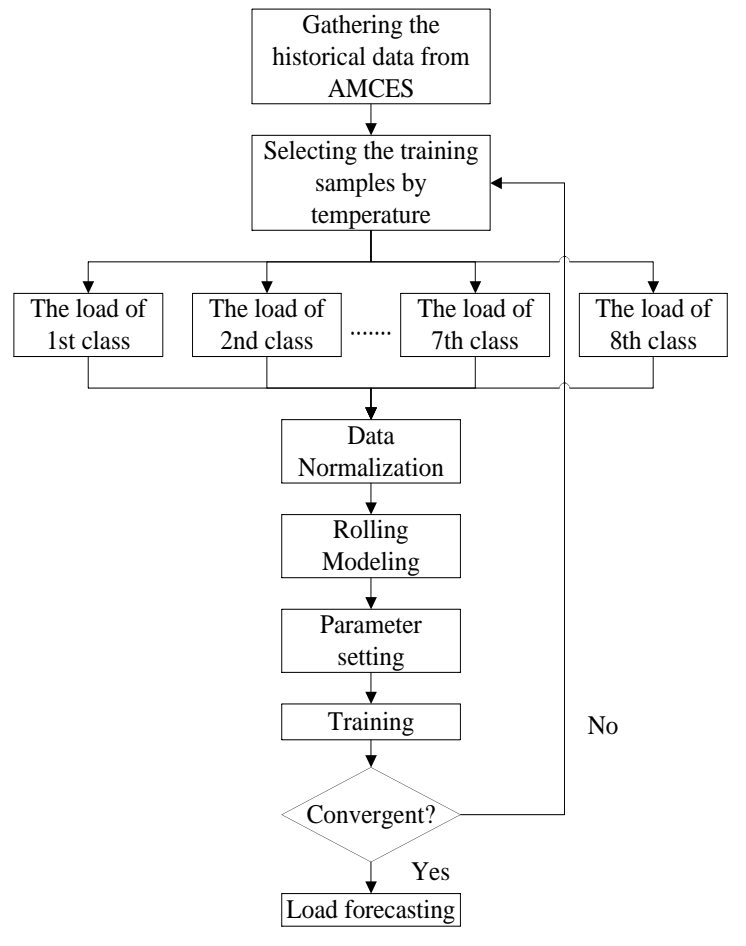

Fig. (7). Proposed flowchart for VSTEDF training by rolling model of BPN.

\section{(1). Training Samples Selection}

The performance of the ANN-based predictor highly depends on the selection of training samples. The training data should cover the entire expected input space. If the training data set has internal consistency, the trained network may produce excellent results. Hence, it is necessary to provide proper extensive types of training samples and proper noise for decreasing the sameness (Liaw 1994) [15]. Hence, in the application of VSTEDF, the training data should be classified according to the data pattern, and the ANN model should be well trained by different day types in order to increase the accuracy of prediction. From the pattern of the usage of electricity, we specified our training inputs into two groups of load trend: the class schedules and the daily peak temperature in this study.

\section{- By Class Hour}

The profile of electric load consumption at NKFUST is correlated closely with the class schedule (see Fig. 8). Generally, the electric load in the first class hour is much lower than others because not many classes start at 8 o' clock in the morning. In order to eliminate the confounding training process of ANN, we grouped the training data into eight class hours per day, beginning at 8:00 in the morning and ending at 5:00 in the evening.

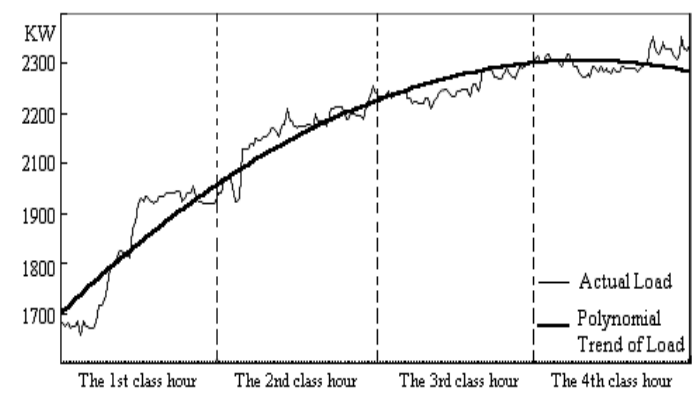

Fig. (8). Load trend is varied by the school class hours at NKFUST.

\section{- By Daily Peak Temperature}

In our preliminary study, we ignored the temperature as defining the input pattern of the network. As a result, the prediction was not optimal (see Fig. 9). As can be seen the forecasted result in Forecast- 2 is better than the one in the Forecast-1. In Forecast-1, the training data with an average temperature of 28.9 which is higher than the daily peak temperature of 21.4. In contrast, in the Forecast-2, the averaged temperature of the training days was about 23.5 which has similar temperature pattern of the daily peak. From this we can conclude that the inputs pattern with closer temperature range will give more accurate prediction. Therefore, the pattern of the daily peak temperature has to be carefully considered.

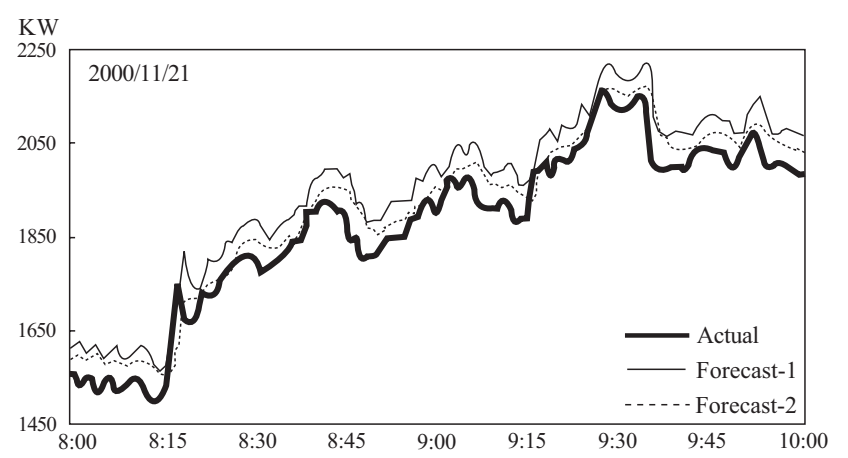

Fig. (9). The daily peak temperature of input patterns affects the performance of prediction. The Forecast- 1 was the prediction with inappropriate inputs pattern of temperature. The Forecast- 2 was the prediction with proper inputs pattern of temperature. The forecasted result of Forecast-2 is better than the one of Forecast-1. 


\section{(2): Data Normalization}

The sigmoidal activation function was chosen as the transfer function of BPN. Therefore, the electric load values should be normalized to the range of 0 to 1 . The calculation formula of normalization is as following:

$$
y=\frac{(H-L)}{(\operatorname{Max}-\operatorname{Min})} \times x+\frac{(\operatorname{Max} \times L-\operatorname{Min} \times H)}{(\operatorname{Max}-\operatorname{Min})}
$$

where

$$
\begin{aligned}
& x: \text { original data } \\
& y: \text { normalized data } \\
& H: \text { top limit of normalization } \\
& \text { L: bottom limit of normalization } \\
& \text { Max: maximum value of original data } \\
& \text { Min: minimum value of original data }
\end{aligned}
$$

\section{(3): BPN Parameter Settings}

There are several learning factors that may affect the BPN training. These factors should be well examined first. In this study, Taguchi's experimental design method was employed to optimize these factors such as (A) number of lagged load points, (B) momentum, (C) number of hidden neurons, and (D) network's learning rate. Table II shows the control factors and their levels. We chose $\mathrm{L}_{9}$ orthogonal

\begin{tabular}{|c|c|c|c|c|c|}
\hline \multirow{2}{*}{ Exp. No. } & \multicolumn{4}{|c|}{ Factors } & \multirow[t]{2}{*}{$\eta$} \\
\hline & $\mathbf{A}$ & B & C & D & \\
\hline 1 & 1 & 1 & 1 & 1 & -7.01 \\
\hline 2 & 1 & 2 & 2 & 2 & -8.63 \\
\hline 3 & 1 & 3 & 3 & 3 & -9.94 \\
\hline 4 & 2 & 1 & 2 & 3 & -10.24 \\
\hline 5 & 2 & 2 & 3 & 1 & -7.01 \\
\hline 6 & 2 & 3 & 1 & 2 & -8.94 \\
\hline 7 & 3 & 1 & 3 & 2 & -8.27 \\
\hline 8 & 3 & 2 & 1 & 3 & -11.10 \\
\hline 9 & 3 & 3 & 2 & 1 & -7.92 \\
\hline
\end{tabular}
array to set up the experimental matrix (see Table $\mathbf{3}$ ).

Table 2. The Control Factors and their Levels in Taguchi's Experimental Design

\begin{tabular}{|l|c|c|c|}
\hline \multicolumn{1}{|c|}{ Control Factors } & Level 1 & Level 2 & Level 3 \\
\hline \hline A Previous load point & 3 & 4 & 5 \\
\hline B Momentum & 0.1 & 0.3 & 0.5 \\
\hline C The number of hidden neurons & 5 & 10 & 15 \\
\hline D Learning rate & 0.1 & 0.3 & 0.5 \\
\hline
\end{tabular}

Table 3. Experimental Matrix and Calculated SN Ratio ( $\eta$ ) Using the $\mathrm{L}_{9}$ Orthogonal Array
Taguchi's method is a quality control method when the system of interest is difficult to model mathematically, or it is cross-linked with quite a few factors and levels. The main advantage of Taguchi's method is that the design of parameters and variance analysis, and the optimal parameters of process can be scientifically analyzed (Walpole 1998) [16]. The most important advantage of Taguchi's method is not the possible reduction of the number of experiments to a minimum, but the ability to represent graphically the combinations of parameters and their $\mathrm{n}$-dimensional forms. The focused factors and their levels for the targeted system have to be decided first in Taguchi's method of experimental design. Taguchi's orthogonal array table is able to quickly collocate the parameter disposition table of focused factors and their levels (see Table 2). Analysis of Variance (ANOVA) of Taguchi's method with the experimental results is then carried out to find out the effect of each factor. The signal-to-noise ratio ( $\mathrm{SN}$ ration) of Taguchi's method is the formation of summary statistics for each combination in the control array. It is used to quickly identify the degree of the influence with the different factor levels and the noise factors that affect the experimental result. That is, the SN ratio is an objective index measuring the stability of quality.

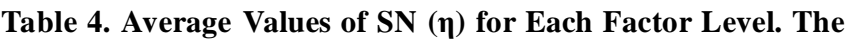
Absolute Values of the Underlined Values are the Smallest Levels for Each Factor. This Combination is the Candidate of the Optimal Combination Set of the Parameters of BPN

\begin{tabular}{|c|c|c|c|c|}
\hline Factor & A & B & C & D \\
\hline \hline 1 & $\underline{-8.53}$ & $\underline{-8.51}$ & -9.02 & $\underline{-7.31}$ \\
\hline 2 & -8.73 & -8.91 & -8.93 & -8.61 \\
\hline 3 & -9.10 & -8.93 & $\underline{-8.41}$ & -10.43 \\
\hline Max. Min. & 0.57 & 0.42 & 0.61 & 3.12 \\
\hline
\end{tabular}

Units: (dB).

Table 5. The DOF, SS, MS, and Contribution in the ANOVA for each factor

\begin{tabular}{|c|c|c|c|c|}
\hline Factor & $\begin{array}{c}\text { Degree of } \\
\text { Freedom }\end{array}$ & $\begin{array}{c}\text { Sum of } \\
\text { Squares }\end{array}$ & $\begin{array}{c}\text { Mean } \\
\text { Square }\end{array}$ & Contribution \\
\hline \hline A & $f_{A}=2$ & $S_{A}=0.5007$ & $V_{A}=0.2504$ & $\rho_{\mathrm{A}}=3.10 \%$ \\
\hline$B$ & $f_{B}=2$ & $S_{B}=0.3478$ & $V_{B}=0.1739$ & $\rho_{\mathrm{B}}=2.15 \%$ \\
\hline$C$ & $f_{C}=2$ & $S_{C}=0.6535$ & $V_{C}=0.3267$ & $\rho_{\mathrm{C}}=4.04 \%$ \\
\hline$D$ & $f_{D}=2$ & $S_{D}=14.671$ & $V_{D}=7.3355$ & $\rho_{\mathrm{D}}=90.71 \%$ \\
\hline Total & $f_{T}=8$ & $S_{T}=16.173$ & & \\
\hline
\end{tabular}

The reacting table of SN, the Signal-to-Noise Ratio ( $\eta$ ) for each factor and level are listed in Table IV. The "smallest-is-the-best" was chosen because accurate prediction of electric load demand is expected. From Table 4, the possible candidates of optimal parameters combination highlighted in the table are $A_{1} B_{1} C_{3} D_{1}$. However, from the ANOVA in 
Table 5, the contributions of factors $\mathrm{A}, \mathrm{B}$ and $\mathrm{C}$ are very little. Therefore, the level of factor $\mathrm{C}$ has to be adjusted from 3 to 1 in order to improve the performance. After the adjustment, the final optimal parameters combination becomes $\mathrm{A}_{1} \mathrm{~B}_{1} \mathrm{C}_{1} \mathrm{D}_{1}$ as shown in Table 2 . That is, the number of lagged load points is 3 ; the momentum is 0.1 ; the number of hidden neurons is 5 ; the learning rate is 0.1 .

\section{RESULTS AND DISCUSSION}

\section{Testing Results}

After the training process by adequate input patterns, we used the trained BPN model to examine our electric load demands. Figs. 10 to $\mathbf{1 2}$ show the forecasted demand electric loads for the regular school days (Monday to Friday), weekend, and school winter recess days respectively. The average absolute errors for general school days, weekend, and winter break days are $2.14 \%, 3.14 \%$, and $1.98 \%$ respectively. The forecasted results show more accurate. Fig. 13 shows the frequency distribution of forecasting error for this test run. Among these predicted points, the errors of more than $55 \%$ forecasted values are smaller than $\pm 2 \%$; and only about $7.28 \%$ are greater than $\pm 5 \%$ error. That is, the predicted results are very satisfactory.

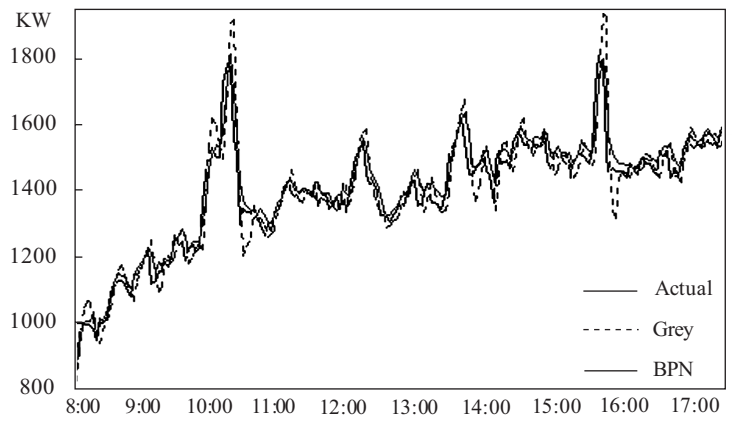

Fig. (10). Actual load vs. forecasted load of general school days (Monday to Friday). It shows accurate predictions.

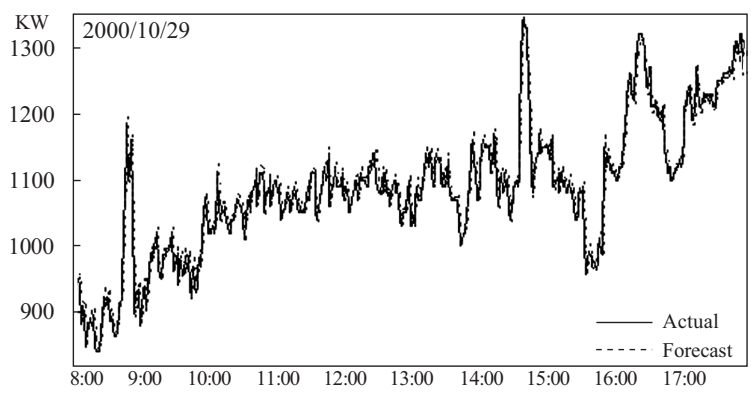

Fig. (11). Actual load vs. forecasted load of weekend. The predictions are accurate and satisfactory.

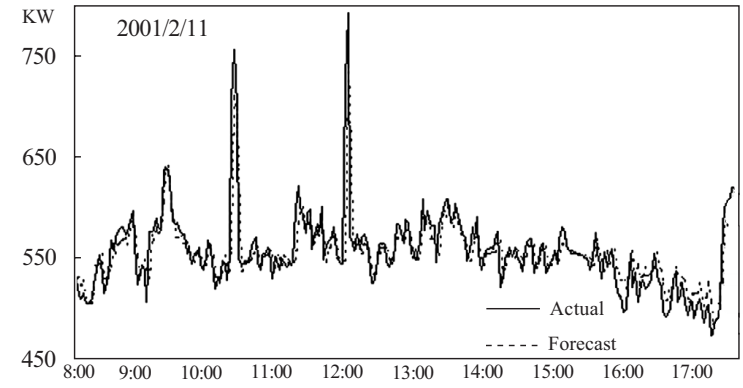

Fig. (12). Actual and forecasted loads of school winter recess. It also shows accurate prediction.

\section{Discussion}

The analysis of the frequency distribution of prediction errors (Fig. 13) and the distribution diagram of actual loads versus forecasted loads (Fig. 14) show clearly that the presented Taguchi-ANN-based forecaster is adequate for VSTEDF of general school days. But in the condition of sudden change of load, the forecasted value was not satisfactory because of the unexpected fluctuation. As shown in Fig. 15, the 94th point of load rocketed up suddenly; the error rose up to $12.76 \%$. However, the prediction for the next point (the 95th point) normalized again quickly. The error decreased to $1.64 \%$ sharply. This unexpected result would not whittle down the value of this presented Taguchi-ANN-based load demand predictor. Because that the forecasting interval is every 2 minutes. The Taiwan Power Company records the over-demand-value load for an interval of every 15 minutes. Therefore, this type of discrepancy will be compensated for quickly in the next 2 minutes.

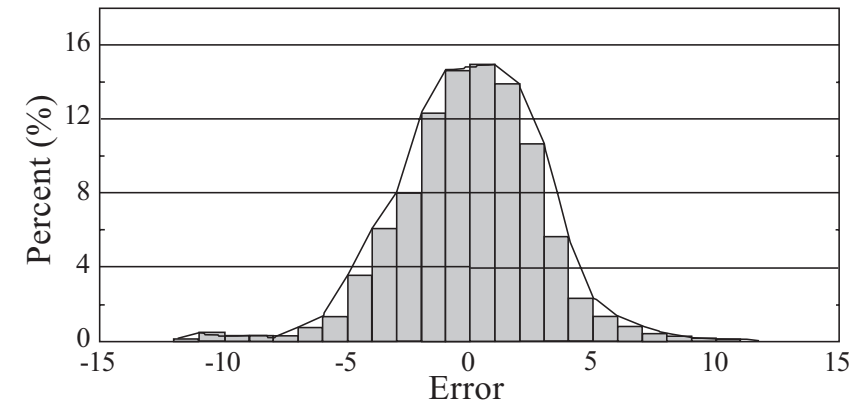

Fig. (13). Forecasting error frequency distribution chart. The errors of more than $55 \%$ forecasted values are smaller than $\pm 2 \%$, and only about $7.28 \%$ are greater than $\pm 5 \%$ error.

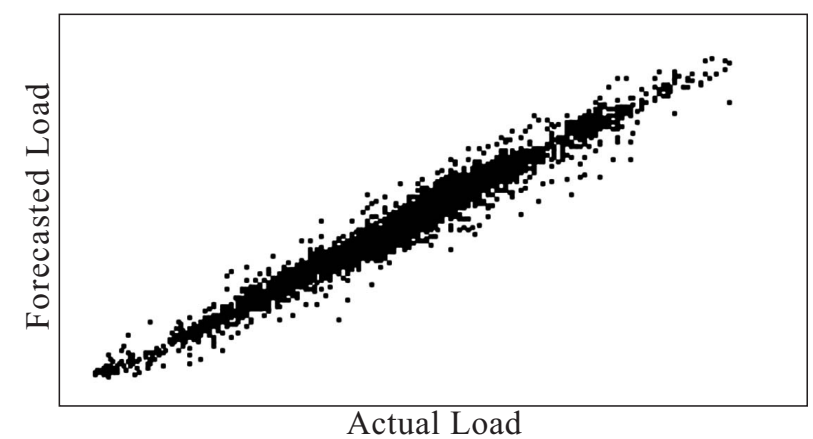

Fig. (14). Distribution diagram of actual load versus forecasted load. It shows the forecasted values are accurate and satisfactory.

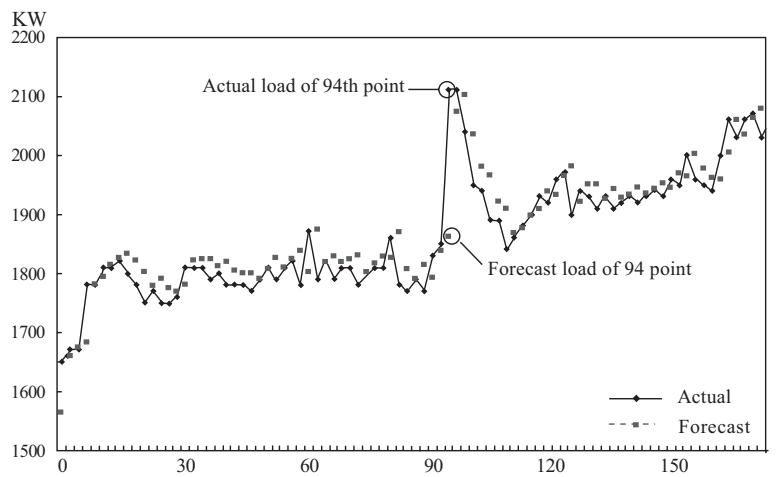

Fig. (15). Diagram of load profile with sudden change at the $94^{\text {th }}$ point. The accuracy of prediction at the sudden change point is dropped dramatically. But it will recover quickly at the next point. 
A brief comparison of the forecasted results with the Grey prediction [14] and BPN is shown in Fig. 16. Since the general Grey model $\operatorname{GM}(1,1)$ is a first-order differential equation varied with time-series. It is defined as $\frac{d X^{(1)}(t)}{d t}+a X^{(1)}(t)=b$, where $a$ and $b$ are Grey parameters Grey prediction model adopts rolling model technique, which utilizes forward data points in the same series to construct Grey model for predicting the next data. The BPN-based load forecasting is a non-linear regression model. The proposed BPN with rolling model technique is like Grey model. However, BPN is a non-linear reflect relation algorithm with multi-layer feed forward network. Therefore the forecasted results of the BPN model are more accurate than the one of Grey prediction.

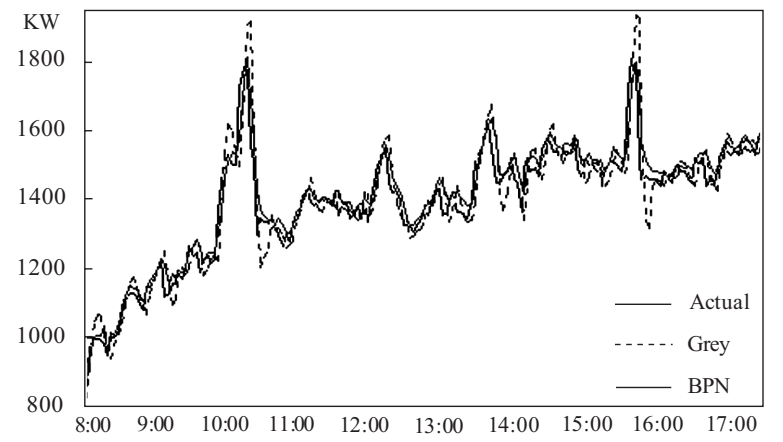

Fig. (16). Comparison between Grey prediction and BPN. The prediction of the rolling model of BPN is better than the one of Grey theory.

\section{CONCLUSIONS}

Instantaneous load forecasting plays an important role for both power plants and consumers today. Power plants need a faster forecasting technique to operate the power systems more reliably and flexibly for optimization strategies. Consumers are able to cut down on electricity bills and avoid penalties with the proposed AMCES and VSTEDF.

In this paper, we present a rolling modeling technique, which is similar to the auto-regression model, to construct the ANN input patterns for very-short-term electric demand forecasting. The rolling modeling technique only needs several historical input variables to construct the ANN model for predicting the electric demands in the interval of every two minutes. At the stage of training samples selection, we specified our training inputs by two types of load trend, the class hours and the daily peak temperature, owing to the patterns of electricity consumption on campus. We also optimized the factors of ANN to obtain a more robust forecasting model by adopting Taguchi's experimental design method.

The experimental results show that the error of forecasting is as small as $3 \%$. Although this model is unable to forecast well when load profile suddenly changes sharply, the error will be compensated for quickly in the next prediction. Our results also show that it is feasible to design a simple and satisfactory dynamic forecaster for predicting very-short-term electric demand by the Taguchi-ANN-based predictor.

\section{ACKNOWLEDGEMENT}

This work was supported in part by the National Science Council of Taiwan under Grant NSC 89-2815-C-327-002-E. The author gratefully acknowledges the contributions of C. T. Yin and C. K. Chen for their works on the original version of this document.

\section{REFERENCES}

[1] Y. H. Fung and V. M. R. Tummala, "Forecasting of electricity consumption: a comparative analysis of regression and artificial neural network models," in Proc. 1993 The 2nd International Conf. on Advances in Power System Control, Operation and Management, vol. 2, pp. 782-787, 1993.

[2] W. Charytoniuk and M. S. Chen, "Very short-term load forecasting using artificial neural networks," IEEE Trans. Power Sys., 15(1): 263-268, 2000.

[3] D. Srinivasan, A. C. Liew, and J. S. P. Chen, "Short term forecasting using neural network approach," in Proc. 1991 The $1^{\text {st }}$ International Forum on Applications of Neural Networks to Power Systems, pp. 12-16, 1991

[4] C. N. Lu, H. T. Wu, and S. Vemuri, "Neural network based short term load forecasting," IEEE Trans. Power Sys., vol. 8(1): 336-342, 1993.

[5] S. J. Kiartzis, A. G. Bakirtzis, and V. Petridis, "Short-term load forecasting using neural networks," Electric Power Sys. Res., 33: $1-6,1995$.

[6] A. Khotanzad, R. Afkhami-Rohani, and D. Maratukulam, "ANNSTLF - artificial neural network based short term load forecaster-generation three," IEEE Trans. Power Sys., 13 (4): 1413-1422, 1998.

[7] T. W. S. Chow, and C. T. Leung, "Neural network based short-term load forecasting using weather compensation," IEEE Trans. Power Sys., 11(4): 1736-1742, 1996.

[8] A. G. Bakirtzis, "A neural network short-term load forecasting model for the Greek power system," IEEE Trans. Power Sys., 11(2): 858-863, 1996.

[9] A. W. L. Yao and C. H. Ku, "Developing a PC-based automated monitoring and control platform for electric power systems," Electric Power Power Sys., 64: 129-136, 2003.

[10] I. Drezga and S. Rahman, "Input variable selection for ANN-based short-term load forecasting," IEEE Trans. Power Sys., 13(4): 1238-1244, 1998.

[11] K. S. Swarup and B. Satish, "Integrated ANN approach to forecast load," IEEE Comput. Appl. Power, 15: 45-51, 2002.

[12] S. Makridakis, S. C. Wheelwright, and R. J. Hyndman, Forecasting Methods and Applications, 3rd ed., New York: John Wiley \& Sons, 1998.

[13] N. K. Bose and P. Liang, Neural Network Fundamentals with Graphs, Algorithms, and Applications, Singapore: McGraw-Hill, 1996, chapter 8.

[14] A.W. L. Yao, S. C. Chi and J. H. Chen, "An improved Grey-based approach for electricity demand forecasting," Electric Power Sys. Res., 67: 217-224, 2003.

[15] P. K. Liaw, "The application of neural network for electric load demand forecasting," Master's thesis, Dept. of Industrial Eng. and Management, Yuan Ze Univ., Chungli, Taiwan, 1994.

[16] R. E. Walpole, R. H. Myers, and S. L. Myers, Probability and Statistics for Engineers and Scientists, New Jersey: Prentice Hall, 1998, chapter 15. 